\title{
Structure and Dynamics of Colloidal Suspensions Studied by Means of XPCS
}

\author{
A. Patkowski ${ }^{a, *}$, J. Gapinski $^{a}$, A. Fluerasu $^{b}$, \\ P. Holmqvist ${ }^{c}$, G. Meier ${ }^{c}$, M.P. Lettinga ${ }^{c}$ And G. NäGele ${ }^{c}$ \\ ${ }^{a}$ Faculty of Physics, A. Mickiewicz University \\ Umultowska 85, 61-614 Poznań, Poland \\ ${ }^{b}$ European Synchrotron Radiation Facility, Grenoble, France \\ ${ }^{c}$ Institut für Festkörperforschung, Forschungszentrum Jülich, Germany
}

Structure and dynamics of suspensions of charged colloidal spheres in water were studied by means of small-angle X-ray scattering and X-ray photon correlation spectroscopy using synchrotron radiation. Experimentally obtained dependences on the scattering vector $q$ of the structure factor $S(q)$, short-time collective diffusion coefficient $D(q)$ and hydrodynamic function $H(q)$ were in good agreement with theoretical predictions. No effect of screening of the hydrodynamic interactions, suggested in the literature, was found. The conditions are discussed at which the maximum of the hydrodynamic function peak value $H\left(q_{\mathrm{m}}\right)$ can be obtained experimentally.

PACS numbers: 87.15.Vv, 83.10.Mj, 82.70.Dd

\section{Introduction}

Colloidal suspensions are heterogeneous systems composed of solid particles or liquid droplets of sizes from $10 \mathrm{~nm}$ to $10 \mu \mathrm{m}$ suspended in a molecular liquid. Suspensions of colloidal particles undergoing Brownian motion are ubiquitous in chemical industry, biology, food science, and in medical and cosmetic products. For polar solvents like water, the particles are usually charged. At long to intermediate distances these particles interact electrostatically by an exponentially screened Coulomb repulsion originating from the overlap of the neutralizing electric double layers. A considerable effort has been devoted over the past years to study the dynamics of colloidal model suspensions of charged spheres at the microscopic level. The dynamics is determined by a subtle interplay of direct interactions and solvent-flow mediated hydrodynamic interaction (HI). HI in unconfined suspensions is very long ranged. It decays with interparticle distance $r$ like $1 / r$. An account of

*corresponding author; e-mail: patkowsk@amu.edu.pl 
HI effects in theoretical and computer simulation studies is quite challenging due to its many-body nature, which must be accounted for in nondilute suspensions. An important measure of the strength of $\mathrm{HI}$ with regard to short-time particle diffusion caused by local density gradients is given by the hydrodynamic function $H(q)$.

The development of partly coherent X-ray beams at synchrotron radiation sources allows for the measurements of the collective translational diffusion coefficient of colloids using the new method of X-ray photon correlations spectroscopy (XPCS) $[1,2]$. Thus, in combination with the small-angle X-ray scattering (SAXS) the scattering vector $(q)$ dependent functions: the structure factor $S(q)$, collective diffusion coefficient $D(q)$ and the hydrodynamic function $H(q)$ can be determined for colloidal suspensions [3, 4]. Recently, using a proper combination of light and $\mathrm{X}$-ray synchrotron radiation scattering, diffusion in suspensions of hard spheres [5-7], charge stabilized spheres [8-11], attractive spheres [12], soft microgel spheres [13], charge spherical proteins [14], and charged and neutral liposomes [15] was measured.

We present a summary of experimental studies of diffusion properties in suspensions of monodisperse charge-stabilized fluorinated latex spheres in water $[3,4]$. Using the synchrotron radiation at ESRF, Grenoble, the SAXS and XPCS studies have been performed to explore in great detail the concentration and ionic-strength dependence of the static structure factor $S(q)$, and of diffusion properties including the wave number-dependent hydrodynamic function $H(q)$, the collective diffusion coefficient $D(q)$, and the intermediate scattering function $S(q, t)$.

We show that all experimental data can be quantitatively described and explained by means of rescaled mean-spherical approximation (RMSA), RogersYoung (RY), and $\delta \gamma$ theories. In particular, the behavior of $H(q)$ for de-ionized dense suspensions can be attributed to the influence of many-body hydrodynamics, without any need to assume hydrodynamic screening to be present in de-ionized fluid systems, as proposed in earlier work [11]. In this work [11] the authors have reported experimental values of the hydrodynamic function well below the hard-sphere limit, for low-salt charge-stabilized suspensions. Such a hindrance (screening) of hydrodynamic interactions cannot be explained by any theoretical model. Thus, the confirmation and validation of this unexpected result is of crucial importance for the understanding and theoretical description of the dynamics of charge-stabilized colloidal suspensions. The obtained values of the maximum of the hydrodynamic function $H\left(q_{\mathrm{m}}\right)$ for all colloid and salt concentrations in the fluid phase were within the general boundaries provided for the magnitude of the hydrodynamic function in the limits of low and high salinity.

The effect of the hydrodynamic interactions in strongly interacting systems is not intuitively predictable. Their most spectacular feature is the acceleration of collective diffusion $(H(q)>1)$. This is in clear contradiction to the hard sphere systems, where hydrodynamic interactions always slow down the diffu- 
sion of colloidal particles - the more the higher the volume fraction. A nonmonotonic behavior of the hydrodynamic function illustrated by its peak value $H\left(q_{\mathrm{m}}\right)$ dependence on the colloid volume fraction $\phi$ can also be found using the $\delta \gamma$ theory [4]. Since some attempts were made to extrapolate tendencies observed in the low volume fraction region to high concentrations (and vice versa), in this work we would like to discuss shortly conditions that have to be met to achieve the maximum value of the hydrodynamic function peak height.

\section{Theoretical}

Static and dynamic properties of suspensions of charged colloidal spheres can be calculated using one-component macroion fluid (OMF) model. In this model colloids are approximated by uniformly charged hard spheres which interact by effective pair potential of the Derjaguin-Landau-Verwey-Overbeek (DLVO) type $[16,17]$ :

$$
\frac{u(r)}{k_{\mathrm{B}} T}=L_{\mathrm{B}} Z^{2}\left(\frac{\mathrm{e}^{\kappa a}}{1+\kappa a}\right)^{2} \frac{\mathrm{e}^{-\kappa r}}{r}, \quad r>2 a,
$$

where $Z$ is the effective charge and $a$ the radius of the colloidal particle, $L_{\mathrm{B}}=$ $e^{2} /\left(\varepsilon k_{\mathrm{B}} T\right)$ is the Bjerrum length for a solvent of the dielectric constant $\varepsilon$. For water at room temperature $L_{\mathrm{B}}=0.71 \mathrm{~nm}$. The electrostatic screening parameter for a closed system is

$$
\kappa^{2}=\frac{4 \pi L_{\mathrm{B}}\left(n|Z|+2 n_{\mathrm{s}}\right)}{1-\phi}=\kappa_{\mathrm{ci}}^{2}+\kappa_{\mathrm{s}}^{2},
$$

where $n$ is the number density of the colloid, $n_{\mathrm{s}}$ - the number density of added monovalent salt, and the volume fraction of the colloid is defined as $\phi=(4 \pi / 3) n a^{3}$. $\kappa^{2}$ consists of contributions due to the monovalent counterions $\kappa_{\mathrm{ci}}^{2}$ and monovalent added salt $\kappa_{\mathrm{s}}^{2}$.

The structure of colloidal suspensions can be described by the static structure factor $S(q)$ :

$$
S(q)=1+n \int \mathrm{d}^{3} r \mathrm{e}^{\mathrm{i} q r}[g(r)-1],
$$

where the scattering vector $q=\frac{4 \pi n}{\lambda} \sin \left(\frac{\Theta}{2}\right), n$ is the refractive index of the solution, $\Theta$ - the scattering angle, $\lambda$ - the wavelength of the incident radiation and $g(r)$ is the radial pair correlation function. The quantity $4 \pi n g(r) r^{2} \mathrm{~d} r$ defines the mean number of particles contained in a spherical shell of radius $r$ and thickness $\mathrm{d} r$ around the chosen particle.

$S(q)$ can be measured by means of light scattering (LS) or SAXS. For spherical colloids of the form factor $P(q)$, the $S(q)$ can be calculated from the measured scattered intensity $I(q)$ using a simple relation: $I(q)=P(q) S(q)$.

Theoretically $S(q)$ can be calculated using the OMF model and numerically solving the Ornstein-Zernike equation with the RY and RMSA closure relations [18-20]. 
In order to characterize the dynamics of colloidal suspensions the intensity (2nd order) time autocorrelation function is usually measured in a coherent light scattering-photon correlation spectroscopy (PCS) or XPCS experiment

$$
g^{(2)}(t)=\frac{\langle I(q, 0) I(q, t)\rangle}{\langle I(q, 0)\rangle^{2}},
$$

which is related to the first-order (field) autocorrelation function $g^{(1)}(t)$ by the Siegert relation [21]:

$$
g^{(2)}(t)=1+A\left|g^{(1)}(t)\right|^{2}
$$

where $A$ is a factor depending on the coherence of the incident radiation and detection system. The short-time collective diffusion coefficient of the colloid $D(q)$ can be obtained from the measured $g^{(1)}(t)$ correlation function as a limiting slope of the $\log g^{(1)}(t)$ :

$$
D(q)=-\frac{1}{q^{2}} \lim _{t \rightarrow 0} \frac{\mathrm{d}}{\mathrm{d} t} \ln g^{(1)}(q, t) .
$$

The hydrodynamic function can be calculated as

$$
H(q)=\frac{D(q) S(q)}{D_{0}}
$$

Thus, for an exact estimation of the hydrodynamic function precise measurements of $S(q)$ and $D(q)$ are needed in a broad $q$-range around the 1st maximum of $S(q)$, as well as an independent measurement of the diffusion coefficient at infinite dilution $D_{0}$.

Theoretically, $H(q)$ can be obtained using the $\delta \gamma$ theory [22] which takes into account three-body and higher order hydrodynamic interactions. The only input needed for these calculations is the structure factor $S(q)$ which can be obtained from the RY and RMSA models.

The functions $D(q), H(q)$, and $S(q)$ can be also calculated using accelerated Stokesian dynamics (ASD) computer simulations [23].

\section{Experimental}

\subsection{Experimental methods}

XPCS was developed in the mid-90-ties of last century when in first experiments using coherent synchrotron radiation the feasibility of the method was demonstrated [1,2]. The XPCS measurements were carried out at the European Synchrotron Radiation Facility, Grenoble, at the ID10A beamline. The experimental setup was described elsewhere [24]. The measurements were performed in the $2 / 3$ filling mode at a wavelength of $\lambda=1.55 \AA$. The intensity of the incident beam, limited by a $12 \mu \mathrm{m}$ pinhole located $25 \mathrm{~cm}$ from the sample, amounted to $2 \times 10^{9}$ photons/s at synchrotron current of $200 \mathrm{~mA}$. The detector - a Bicron scintillation counter with effective area of $150 \times 150 \mu \mathrm{m}^{2}$ was located $3.33 \mathrm{~m}$ from the sample. An ALV-5000 digital correlator was used to obtain the correlation functions. SAXS measurements were performed with the same setup. 


\subsection{Sample}

Water suspensions of charged fluorinated latex spheres prepared by soap-free emulsion polymerization were used [25]. The hydrodynamic radius of the spheres measured by means of light scattering-PCS amounted to $80 \mathrm{~nm}$. The radius obtained from the form factor of the SAXS measurements amounted to $(78.5 \pm 1.6) \mathrm{nm}$. In order to decrease the ionic strength the suspension was kept for $10 \mathrm{~h}$ in contact with an ion exchange resin (Amberlite MB3, Sigma). Obtained in this way crystalline suspension was used to prepare two suspensions: high salt at $50 \mathrm{mM}$ and low salt at $0.5 \mathrm{mM}$ added $\mathrm{NaCl}$ concentration. Three different colloid concentration samples were prepared at each salt concentration. The colloid volume fraction of the low salt samples was in the range, where hydrodynamic screening was reported to occur [11]. For the XPCS and SAXS measurements all samples were filled into $1.5 \mathrm{~mm}$ quartz capillaries and sealed with glue.

For simplicity the six samples were coded with a symbol ("S" for "screened" and "NS" for "not screened") and a number denoting the concentration (1 for the highest and 3 for the lowest), so that S-1 will refer to the highest colloid concentration with high salt content and NS-3 to the lowest colloid concentration with low salt content. The actual concentration of the samples was estimated to: NS-1 - 200, NS-2 - 97, NS-3 - 75, S-1 - 231, S-2 - 95, S-3 - 50 mg $/ \mathrm{ml}$. These values corresponded to the volume fractions of: NS-1 -0.123 , NS-2 0.0597 , NS-3 -0.0462 , S- $1-0.142$, S- $2-0.0585$, S-3 -0.0308 .

\section{Results and discussion}

One of the parameters which strongly affects the process of ordering and eventually crystallization of colloidal particles is the polydispersity of the sample. For most of the theoretical models used in the colloidal science, inclusion of polydispersity causes a rapid increase in computational effort and makes the comparison between different systems very difficult, especially in terms of the conditions that should be met to achieve (or avoid) crystallization. For those reasons it is much more convenient to use monodisperse systems. Thanks to the special procedure of synthesizing the latex particles, our sample was very monodisperse. In Fig. 1 we show the SAXS results measured for the sample NS-1 in a broad $q$-range. Since the oscillations of the structure factor $S(q)$ decay quickly even for highly structured samples, to a good approximation the intensity spectrum $I(q)$ beyond $q=0.08 \mathrm{~nm}^{-1}$ reduces to the form factor $P(q)$. Well developed and sharp multiple minima in this spectrum confirm the quality of our sample in terms of monodispersity. The fit of a polydisperse sphere form factor to the experimental data allowed us to estimate the radius of the particles as $78.5 \mathrm{~nm}$ with a $2 \%$ relative standard deviation of the assumed Gaussian distribution. With those many observed minima the accuracy of the radius magnitude was better than $0.1 \mathrm{~nm}$.

For all six samples we first measured the SAXS spectrum to achieve the solution structure factors $S(q)$ and locate the peak positions $q_{\mathrm{m}}$. The data were 


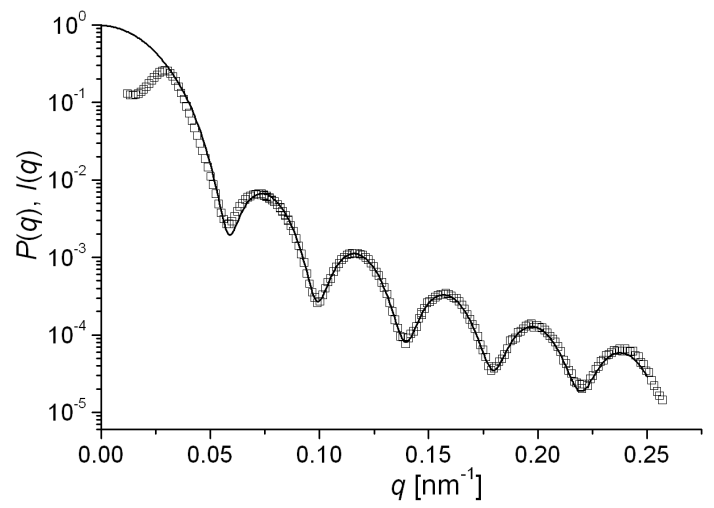

Fig. 1. Scattered X-ray intensity $I(q)$ of the NS-1 sample and fitted form factor $P(q)$ calculated assuming a Gaussian distribution of the sphere radii with the mean value of $78.5 \mathrm{~nm}$ and relative standard deviation of $2 \%$.

fitted with the RMSA model to obtain the information about the effective charge of the colloid particles and the actual volume fraction as a control to the sample preparation procedure. The results of SAXS measurements can be seen in the upper plots of Fig. 2a and b together with the RMSA fits to the experimental data. For the samples with low added salt content the effective charge values $Z_{\text {eff }}$ obtained from the RMSA calculations amounted to: NS-1 - 1600, NS-2 - 1800, NS-3 - $1800 e$, respectively.
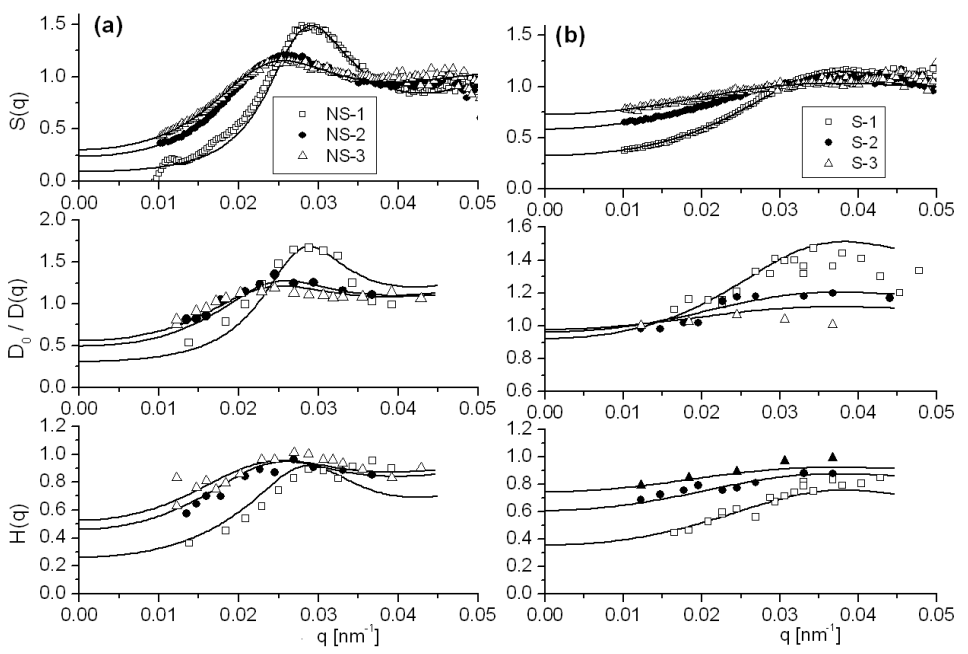

Fig. 2. Experimental results of the structure factor $S(q)$, reciprocal normalized diffusion coefficients $D_{0} / D(q)$ and calculated hydrodynamic function $H(q)$ for the samples with weakly (a) and highly (b) screened electrostatic interactions. The lines represent model predictions fitted to experimental data: RMSA for $S(q)$ and $\delta \gamma$ for $D_{0} / D(q)$. 
The XPCS measurements were performed at several points in the vicinity of $q_{\mathrm{m}}$ to probe the collective dynamics of the colloid particles in the length-scale comparable to the mean interparticle distance. Typical correlation functions are shown in Fig. 3. The diffusion coefficients were estimated from the initial slopes of the $\ln g^{(1)}(t)$ - as in the inset of Fig. 3. The $D_{0}$ value of the diffusion coefficient was estimated from the dynamic light scattering on a very dilute sample with high added salt content (series "S") to $2.69 \times 10^{-8} \mathrm{~cm}^{2} / \mathrm{s}$. The results of the XPCS measurements are shown in the middle plots of Fig. 2a and b in the form of inverse relative values of the diffusion coefficient $D_{0} / D(q)$. For each set of data the $\delta \gamma$ calculations were performed to find a set of model parameters fitting best the measured values. The results of this analysis are presented in Fig. 2a and b as solid lines. The three correlation functions shown in Fig. 3 were measured at three $q$ values: one at the peak of $D_{0} / D(q)$ and one on either side of that peak. As can be seen in Fig. 3 the non-exponentiality of the correlation functions is very weak if any at all in the vicinity of $q_{\mathrm{m}}$.

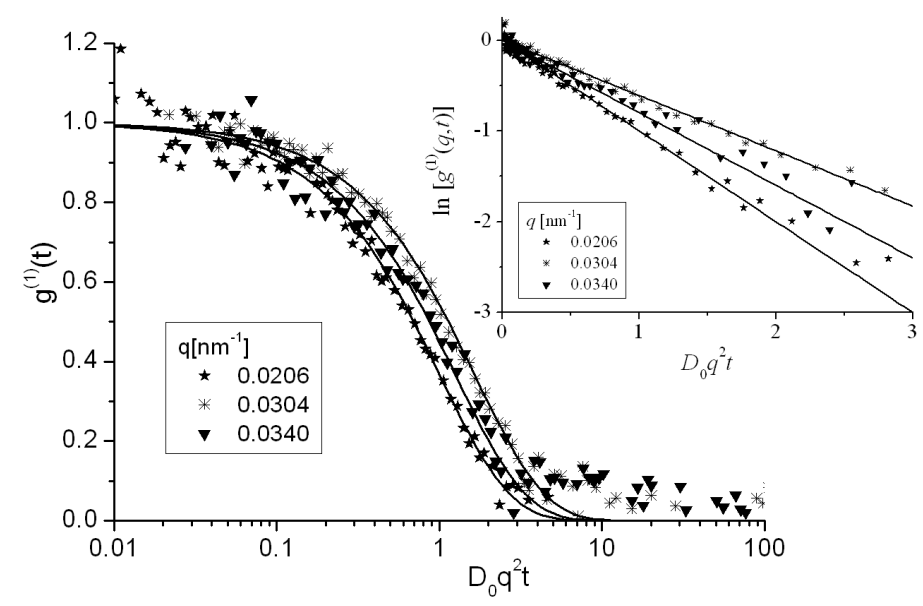

Fig. 3. Normalized first order correlation functions of the X-ray scattered intensity measured for the NS-1 sample at three different angles corresponding to scattering vector values as indicated in the figure legend. The inset shows the traditional ln-lin representation of the same data. The solid lines represent single-exponential fits to the experimental data (initial slopes in the inset).

The hydrodynamic functions $H(q)$ were calculated from the model (fitted) values of $S(q)$ and experimental values of $D(q) / D_{0}$ using Eq. (7). These points are shown in the bottom plots of Fig. 2a and b together with the lines representing the $\delta \gamma$ calculations. For the samples in the "S" series the amount of the added salt was sufficient to reach the hard sphere limit and the measured values nicely followed the theoretical predictions for hard spheres. For the "NS" series the acceleration effect $\left(H\left(q_{\mathrm{m}}\right)>1\right)$ was not observed but the values of $H(q)$ in the vicinity of the 
peak clearly raised above the hard sphere limit. In accordance with the theoretical calculations, $H(q)$ becomes steeper with increasing interaction strength, which can be illustrated by the larger amplitude of $H(q)$ for the samples in the series "NS" as compared to the ones in the "S" series.

As one can see in Fig. 2, the experimental data concerning $S(q), D(q)$, and $H(q)$ are in very good agreement with the RMSA, RY, and $\delta \gamma$ theories and ASD computer simulations. A detailed analysis and discussion of these data was presented earlier [3, 4]. Thus, we can conclude that the previously suggested [11] effect of screening of $\mathrm{HI}$ is not present in our data and theories used so far properly describe the measured quantities.

In Fig. 4 we present the dependence of the hydrodynamic function peak value $H\left(q_{\mathrm{m}}\right)$ on the sample volume fraction $\phi$. The upper broken lines refer to the theoretical predictions in dilute deionized systems. The sublinear dependence on $\phi$ was found by numerical calculations in the limit of two-body hydrodynamic interactions [28]. The lower solid line represent the hard sphere limit obtained using the $\delta \gamma$ model. The points in the plot represent experimental data and simulation results. Apart from our "NS" series data, all available experimental points refer to the hard sphere-like systems, including our "S" series. Only simulation results can be found in the space between the two limits. In order to find experimental conditions which would allow to fill this gap, we performed some extended calculations for our system using the $\delta \gamma$ model. We assumed almost salt free environment $\left(10^{-7} \mathrm{M}\right)$ and calculated the structure factor $S(q)$ and the hydrodynamic function $H(q)$ in the vicinity of the structure peak for a series of volume fractions ranging from 0.0001 to 0.4 using different values of the effective charge $Z_{\text {eff }}$. The value of $S\left(q_{\mathrm{m}}\right)=3.5$ signaling the crystallization, was used as a cut-off criterion for utilizing the calculated parameters: all of the results with $S\left(q_{\mathrm{m}}\right)>3.5$ were removed from further analysis.

In Fig. 5 we plotted calculated peak values of $H(q)$ as a function of the sample volume fraction. The lines correspond to constant $Z_{\text {eff }}$ values as indicated in the figure caption. The range of calculations for each $Z_{\text {eff }}$ was limited by the crystallization criterion $S\left(q_{\mathrm{m}}\right)=3.5$. For each line presented in Fig. 5 we read the maximum values of $H\left(q_{\mathrm{m}}\right)$ and the volume fractions at which this maximum was found. The results of this procedure are presented in Fig. 6.

From the plot of the maximum value of $H\left(q_{\mathrm{m}}\right)$ as a function of $Z_{\text {eff }}$ (Fig. 6a) we can see that it increases with increasing interaction strength as long as the sample remains liquid. For colloidal particles of the size equal to the system reported in this work the highest value of $H\left(q_{\mathrm{m}}\right)$ is about 1.30 , provided that the particles will have sufficient effective charge (about $500 \mathrm{e}$ ). It is interesting to note that the volume fractions at which $H\left(q_{\mathrm{m}}\right)$ are significantly greater than one are rather small: $5.5 \%$ at the most (see Fig. $6 \mathrm{~b}$ ). Similar observations have been made for simulations on another system ( $a=50 \mathrm{~nm}$ in deionized water [4]) in our earlier work. 


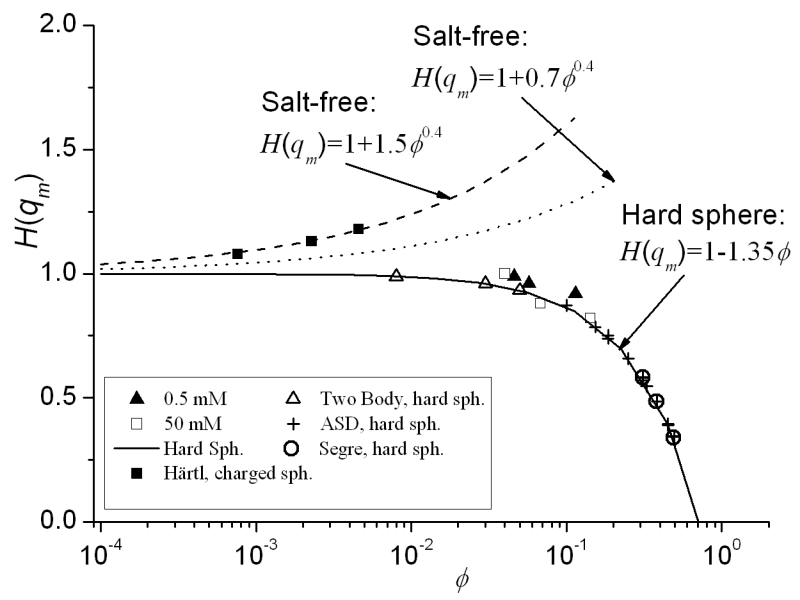

Fig. 4. Maximum of $H(q)$ as function of colloid volume fraction $\phi$. Dashed lines: deionized suspensions (with experimental data of Härtl et al. [26] (-) overlayed). Solid line: hard spheres. Further included are our experimental data for $0.5 \mathrm{mM}(\boldsymbol{\Delta})$ and $50 \mathrm{mM}(\square)$ of added $\mathrm{NaCl}$. Additionally displayed are dynamic light scattering data of Segre et al. [27] (०), low-density calculation results with full 2-body HI included [28] $(\triangle)$, and ASD results [29] $(+)$ for colloidal hard spheres.

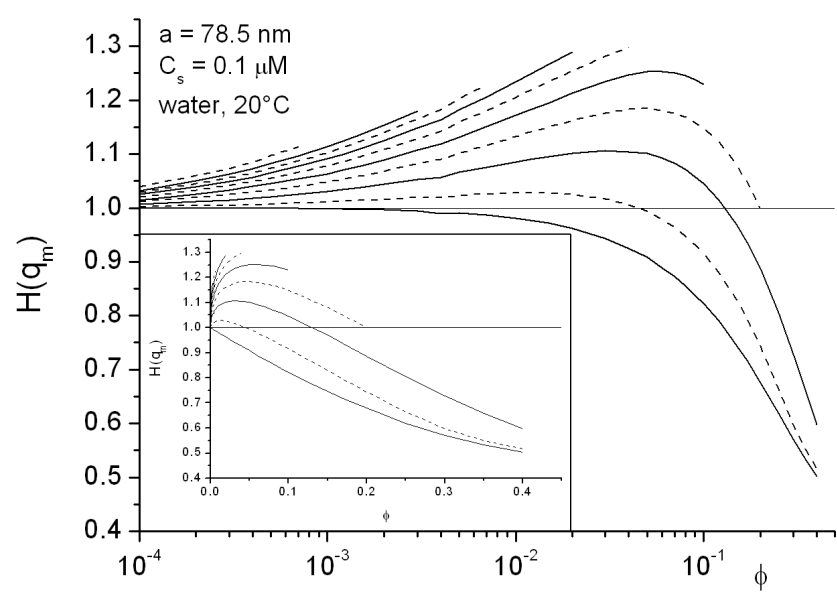

Fig. 5. Peak values of the hydrodynamic function $H(q)$ calculated using the $\delta \gamma$ model for an almost salt-free $(0.1 \mu \mathrm{M})$ system of homogeneous spheres with the radius of $a=78.5 \mathrm{~nm}$ in water as a function of the volume fraction $\phi$. The lines represent the results obtained for the following effective charge values (bottom to top): 0, 100, 200, $300,400,500,600,700,800,1000 \mathrm{e}$. The inset shows the same data in the lin-lin representation. 

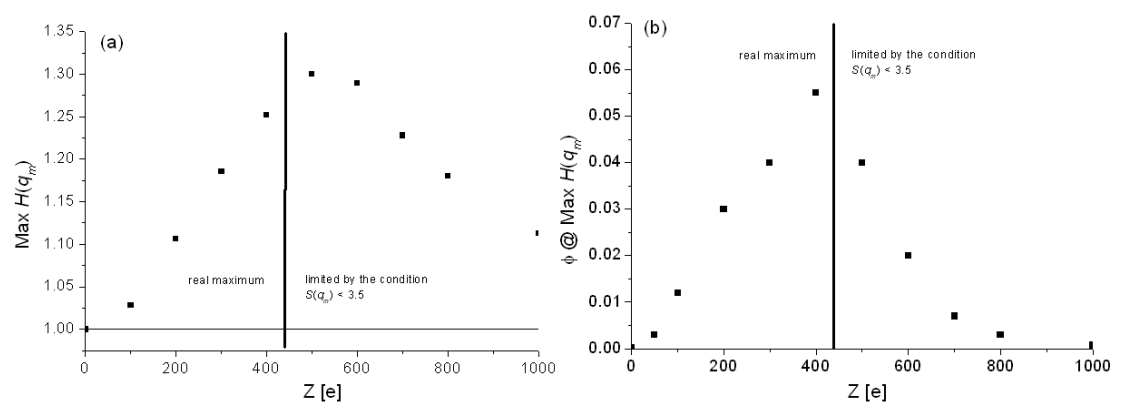

Fig. 6. Maximum value of hydrodynamic function peak heights as a function of effective charge used in $\delta \gamma$ calculations (a) and the volume fraction at which this maximum is achieved (b). The plots refer to the system presented in Fig. 5. The thick vertical lines show the conditions where the maximum value of $H\left(q_{\mathrm{m}}\right)$ is limited by the crystallization criterion.

It seems that the effect of collective diffusion acceleration $(H(q)>1)$ can be observed when two conditions are met simultaneously: (i) the system is highly ordered (sharp peak in $S(q)$ ), (ii) the particles are relatively far away from one another with respect to their radii (low volume fractions). The first condition is required to create the colloid radial distribution responsible in some way for the acceleration effect. The second one is obligatory because when the particles are too close to one another the hydrodynamic hindrance eventually slows down all kinds of motion. In the case of our system, the mean interparticle distance in the conditions where $H\left(q_{\mathrm{m}}\right)=1.30$ was equal to $2.12 \times(2 a)$ which means that the mean distance between the surfaces of neighboring particles was $12 \%$ larger than their diameter.

\section{Conclusions}

Our recent studies $[3,4]$ were devoted to systems of monodisperse charged colloidal particles in water with well controlled amounts of added salt. Combined measurements of static and dynamic X-ray scattering allowed us to determine the $q$-dependence of the static structure factors $S(q)$ and the collective diffusion coefficients $D(q)$ at different colloid volume fractions and added salt concentrations which determined the interaction strength. The hydrodynamic functions $H(q)$ calculated from these data could be well described using the appropriate theoretical models $(\delta \gamma)$ and no signature of hydrodynamic screening was found as reported by other authors [11]. Calculations of the maximum values of the hydrodynamic function $H\left(q_{\mathrm{m}}\right)$ in a broad range of $\phi$ and $Z_{\text {eff }}$ revealed a characteristic region in the $\left(\phi, Z_{\text {eff }}\right)$ plane (Fig. 6) where all the values of $H\left(q_{\mathrm{m}}\right)$ corresponding to different combinations of $\left(\phi, Z_{\text {eff }}\right)$ can be found. This type of analysis will help in the interpretation of old experimental data and prediction of conditions where hydrodynamic acceleration or hindrance may be found in the investigated colloidal system. 


\section{Acknowledgments}

The work has been supported in part by the European Network of Excellence 'SoftComp', Project No. S080118.

\section{References}

[1] T. Albrecht, W. Steffen, A. Patkowski, G. Meier, E.W. Fischer, G. Grübel, D.L. Abernathy, Phys. Rev. Lett. 77, 5437 (1996).

[2] S.B. Dierker, R. Pindak, R.M. Fleming, I.K. Robinson, L. Berman, Phys. Rev. Lett. 75, 449 (1995).

[3] A.J. Banchio, J. Gapinski, A. Patkowski, W. Haeussler, A. Fluerasu, S. Saccana, P. Holmqvist, G. Meier, M.P. Lettinga, G. Naegele, Phys. Rev. Lett. 96, 138303 (2006).

[4] J. Gapinski, A. Patkowski, A.J. Banchio, P. Holmqvist, G. Meier, M.P. Lettinga, G. Nägele, J. Chem. Phys. 126, 104905 (2007).

[5] D. Lumma, L.B. Lurio, M.A. Borthwick, P. Falus, S.G.J. Mochrie, Phys. Rev. E 62, 8258 (2000).

[6] P.N. Pusey, P.N. Segre, O.P. Behrend, S.P. Meeker, W.C.K. Poon, Physica A 235, 1 (1997).

[7] P.N. Segre, P.N. Pusey, Phys. Rev. Lett. 77, 771 (1996).

[8] L.F. Rojas, R. Vavrin, C. Urban, J. Kohlbrecher, S. Stradner, F. Scheffold, P. Schurtenberger, Faraday Discuss. 123, 385 (2003).

[9] M. Tirada-Miranda, C. Haro-Perez, M. Quesada-Perez, J. Callejas-Fernandez, R. Hidalgo-Alvarez, J. Colloid Interface Sci. 263, 74 (2003).

[10] E. Overbeck, C. Sinn, M. Watzlawek, Phys. Rev. E 60, 1936 (1999).

[11] D.O. Riese, G.H. Wegdam, W.L. Vos, R. Sprik, D. Fenistein, J.H.H. Bongaerts, G. Grübel, Phys. Rev. Lett. 85, 5460 (2000).

[12] K.F. Seefeldt, M.J. Solomon, Phys. Rev. E 67, 050402 (2003).

[13] S. Pyett, W. Richtering, J. Chem. Phys. 122, 034709 (2005).

[14] J. Gapinski, A. Wilk, A. Patkowski, W. Häußler, A.J. Banchio, R. Pecora, G. Nägele, J. Chem. Phys. 123, 054708 (2005).

[15] C. Haro-Perez, M. Quesada-Perez, J. Callejas-Fernandez, E. Casals, J. Estelrich, R. Hidalgo-Alvarez, J. Chem. Phys. 119, 628 (2003).

[16] W.B. Russel, D.A. Saville, W.R. Schowalter, Colloidal Dispersions, Cambridge University Press, Cambridge 1989.

[17] A.R. Denton, Phys. Rev. E 62, 3855 (2000).

[18] F.J. Rogers, D.A. Young, Phys. Rev. A 30, 999 (1984).

[19] J.P. Hansen, J.B. Hayter, Mol. Phys. 46, 651 (1982).

[20] G. Nägele, The Physics of Colloidal Soft Matter, Lecture Notes, Vol. 14, Institute of Fundamental Technological Research, Polish Academy of Sciences, Warsaw 2004 .

[21] B.J. Berne, R. Pecora, Dynamic Light Scattering, Wiley, New York 1976.

[22] C.W.J. Beenakker, P. Mazur, Physica (Amsterdam) 126A, 349 (1984). 
[23] A.J. Banchio, J.F. Brady, J. Chem. Phys. 118, 10323 (2003).

[24] T. Thurn-Albrecht, F. Zontone, G. Grübel, W. Steffen, P. Müller-Buschbaum, A. Patkowski, Phys. Rev. E 68, 031407 (2003).

[25] G.H. Koenderink, S. Sacanna, C. Pathmamanoharan, M. Rasa, A.P. Philipse, Langmuir 17, 6086 (2001).

[26] W. Härtl, Ch. Beck, R. Hempelmann, J. Chem. Phys. 110, 7070 (1999).

[27] P.N. Segre, O.P. Behrend, P.N. Pusey, Phys. Rev. E 52, 5070 (1995).

[28] A.J. Banchio, G. Nägele, J. Bergenholtz, J. Chem. Phys. 111, 8721 (1999).

[29] A.J. Banchio, G. Nägele, unpublished results. 